УДК 004.85

\title{
ИССЛЕДОВАНИЕ ПРИМЕНИМОСТИ МЕТОДА ГРАДИЕНТНОГО БУСТИНГА ДЛЯ РЕШЕНИЯ ЗАДАЧИ ПРОГНОЗИРОВАНИЯ
}

\author{
Хлебородова Лидия Дмитриевна \\ студент
}

Научный руководитель: Осипов Геннадий Сергеевич

д.т.н., зав. кафедрой Информатики ФГБОУ ВО «Сахалинский государственный университет»

\begin{abstract}
Аннотация: Отработана концептуальная основа принципов решения задачи прогнозирования на больших данных методом градиентного бустинга в среде пакета Wolfram Mathematica. Проведен анализ возможности понижения размерности задачи без существенной потери информационной емкости обучающей выборки с целью снижения вычислительной трудоемкости используемого метода.
\end{abstract}

Ключевые слова: прогнозирование, машинное обучение, градиентный бустинг.

\section{INVESTIGATION OF THE APPLICABILITY OF THE GRADIENT BOOSTING METHOD FOR SOLVING THE FORECASTING PROBLEM}

\section{Khleborodova Lidiia Dmitrievna Scientific adviser: Osipov Gennady Sergeevich}

\begin{abstract}
The conceptual basis of the principles of solving the forecasting problem on big data by the gradient boosting method in the environment of the Wolfram Mathematica package has been worked out. The analysis of the possibility of reducing the dimension of the data without a significant loss of the information capacity of the training sample in order to reduce the computational complexity of the method used.
\end{abstract}

Key words: forecasting, machine learning, gradient boosting. 


\section{Обучающая выборка}

Исходными данными для задачи является большой массив данных о наблюдаемых параметрах, характеризующих поведение сложного социальноэкономического объекта.

На рисунке 1 представлен фрагмент обучающей выборки [1], в которой $m=1002$ результата наблюдений, $n=6$ входных переменных: $x=\left\{x_{1}, x_{2}, ., x_{6}\right\}$ и одна выходная переменная $y$.

\begin{tabular}{||l|l|l|l|l|l|l|}
\hline \multicolumn{1}{|c|}{$\mathbf{x 1}$} & \multicolumn{1}{c}{$\mathbf{x 2}$} & \multicolumn{1}{c}{$\mathbf{x 3}$} & $\mathbf{1 4}$ & \multicolumn{1}{c|}{$\mathbf{x 5}$} & \multicolumn{1}{c|}{$\mathbf{x 6}$} & \multicolumn{1}{c|}{$\mathbf{y}$} \\
\hline 243,99 & 244,74 & 241,06 & 243,25 & 2541960 & 617617305,1 & 243,73 \\
\hline 244,8 & 246,9 & 243,5 & 243,73 & 2966460 & 727341922,9 & 242,65 \\
\hline 243,52 & 244,72 & 242,11 & 242,65 & 2712340 & 659072971,4 & 240,82 \\
\hline 242 & 242,64 & 240,03 & 240,82 & 2103330 & 507203050,3 & 240,87 \\
\hline 241,75 & 243,5 & 239,15 & 240,87 & 2594210 & 626355852,6 & 239,1 \\
\hline 241,18 & 242,28 & 238,38 & 239,1 & 2936390 & 703924933,3 & 236,1 \\
\hline 239,87 & 241,37 & 236 & 236,1 & 3474100 & 826500817,5 & 234,5 \\
\hline 236,78 & 239,3 & 234,1 & 234,5 & 5514040 & 1301159033 & 238,18 \\
\hline 236 & 239,34 & 236 & 238,18 & 4222420 & 1004241050 & 237,6 \\
\hline 240,57 & 241,27 & 236,22 & 237,6 & 3323780 & 794011671,7 & 238,91 \\
\hline
\end{tabular}

Рис. 1 Фрагмент обучающей выборки

\section{Постановка задачи}

Объектом и предметом исследования является проблема построения отображения $f: x \rightarrow y$, где $x$ - вектор внутренних аргументов; $y$ прогнозируемая величина.

Цель исследования - отработка методологии синтеза искомого отображения $f$ методом извлечения новых знаний из базы данных с помощью алгоритма градиентного бустинга, а также исследование возможности снижения размерности задачи без потери информационной емкости исходных данных.

\section{Метод решения}

Для решения поставленной задачи применялся метод градиентного бустинга на базе деревьев решений (Gradient Boosted Trees) [2, 3].

Основные критерии оценки качества прогноза приведены в таблице 1. 
Таблица 1

\section{Критерии оценки качества прогноза}

\begin{tabular}{|l|l|l|}
\hline \multirow{2}{*}{$\begin{array}{l}\text { Средние } \\
\text { оценки }\end{array}$} & $\begin{array}{l}\text { Среднеквадратичная ошибка (Root } \\
\text { Mean Square Error) }\end{array}$ & $\mathrm{RMSE}=\sqrt{\frac{\sum_{i=1}^{m}\left(y_{i}-\tilde{y}_{i}\right)^{2}}{m}}$ \\
\cline { 2 - 3 } & $\begin{array}{l}\text { Средняя процентная ошибка (Mean } \\
\text { Absolute Percentage Error) }\end{array}$ & $\mathrm{MAPE}=\frac{1}{m} \sum_{i=1}^{m} \frac{\left|y_{i}-\tilde{y}_{i}\right|}{y_{i}} \cdot 100 \%$ \\
\hline $\begin{array}{l}\text { Точечная } \\
\text { оценка }\end{array}$ & $\begin{array}{l}\text { Ошибка экстраполирования } \\
\text { (Relative Extrapolation Error) }\end{array}$ & $\mathrm{REE}=\frac{\left|y_{m+1}-\tilde{y}_{m+1}\right|}{y_{m+1}} \cdot 100 \%$ \\
\hline
\end{tabular}

здесь $y$-известное (табличное) значение выходной переменной,

$\tilde{y}$ - расчетное (предсказанное) значение.

Компьютерное моделирование и практическая апробация синтезируемой системы прогнозирования на больших данных выполнялись на базе аналитической платформы Wolfram Mathematica [3, 4], ориентированной на выполнение символьной обработки информации, характерной для машинного обучения систем искусственного интеллекта.

\section{Основные результаты}

На рисунке 2 приведен оператор построения модели прогнозирования методом градиентного бустинга и приведена информация о размерности обучающей выборки, т.е. количестве $n$ учитываемых входных переменных, а также о количестве $m$ обучающих образов (примеров).

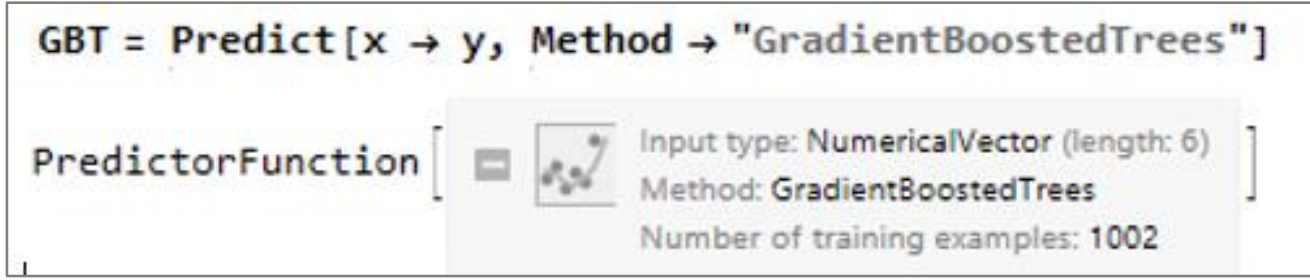

\section{Рис. 2. Построение модели}

Рисунок 3 дает полную характеристику процесса обучения модели, включая изменение во времени стандартного отклонения. 


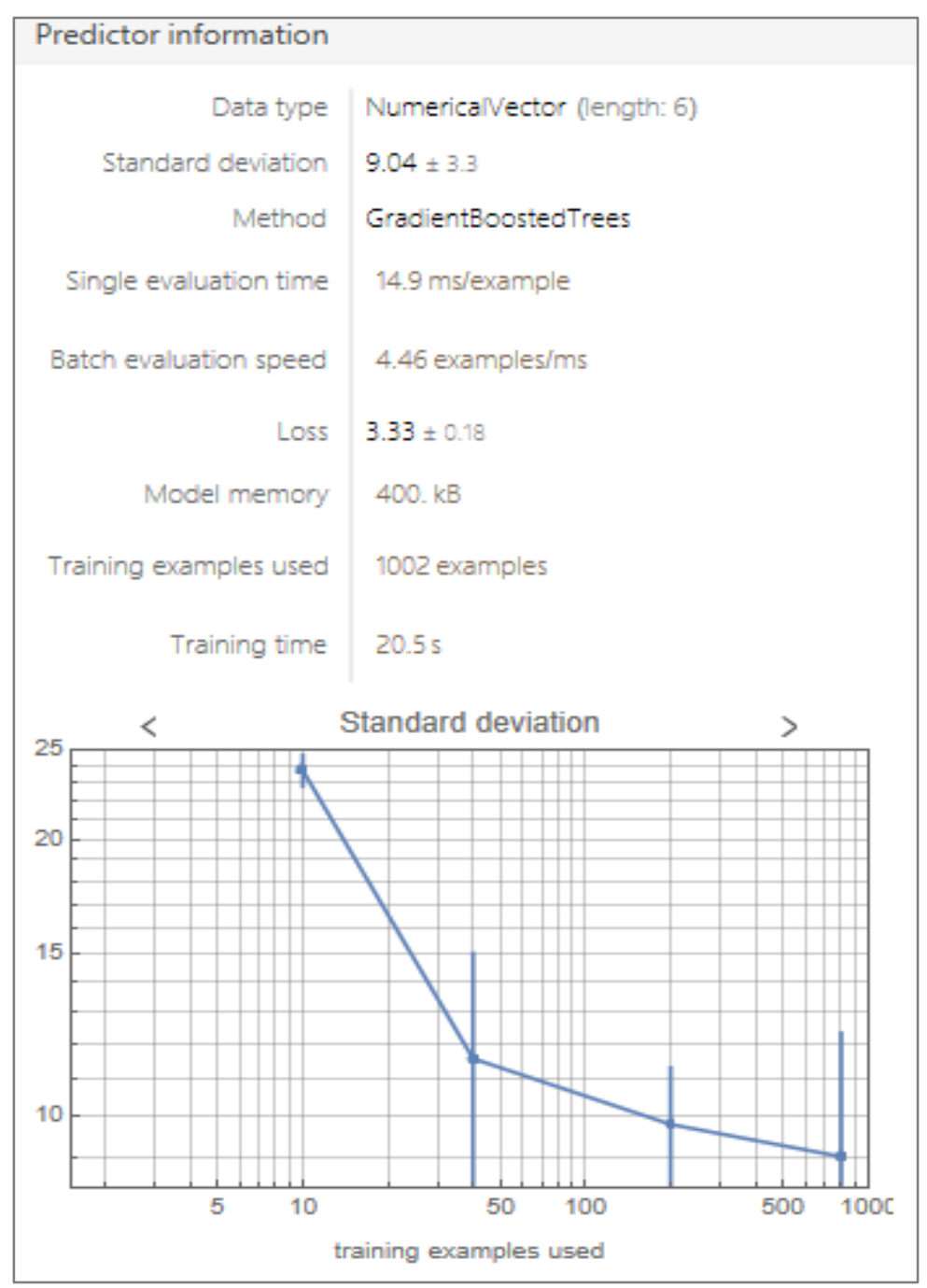

\section{Рис. 3. Иллюстрация процесса построения модели прогноза}

На рисунке 4 представлен фрагмент программы, обеспечивающий расчет показателей качества обучения модели.

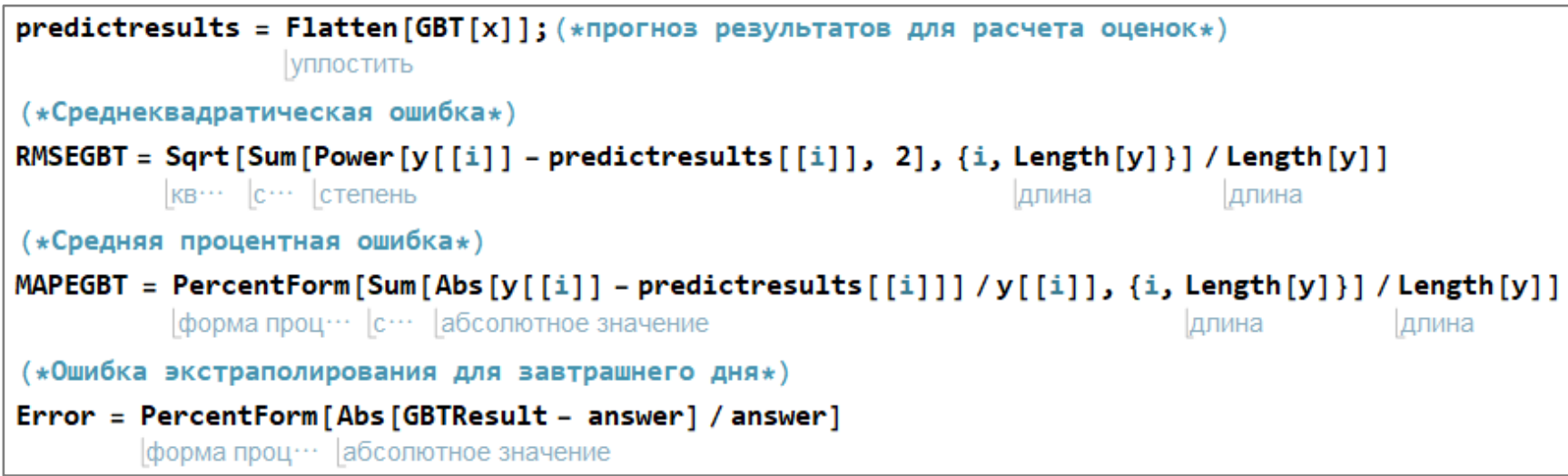

Рис. 4. Расчет критериев качества обучения 
Из исходных $n=6$ входных переменных две в данном случае имеют несущественный коэффициент корреляции с результатом прогноза, поэтому они были исключены из модели. Затем, проводилось поэтапное понижение размерности (методом главных компонент) от 4 до 1.

Основные результаты исследования влияния размерности задачи на качество прогнозирования в соответствии с выбранными оценками сведены в таблицу 2.

\section{Таблица 2}

Основные оценки качества прогноза

\begin{tabular}{|l|c|c|c|c|c|}
\hline \multirow{2}{*}{ Ошибка } & \multicolumn{5}{|c|}{$n$} \\
\cline { 2 - 6 } & 6 & 4 & 3 & 2 & 1 \\
\hline RMSE & 3.672 & 3.886 & 4.007 & 3.898 & 5.031 \\
\hline MAPE & $1.03 \%$ & $1.09 \%$ & $1.09 \%$ & $1.02 \%$ & $1.38 \%$ \\
\hline REE & $0.33 \%$ & $0.55 \%$ & $1.12 \%$ & $0.35 \%$ & $0.45 \%$ \\
\hline
\end{tabular}

Из данных, приведенных в таблице 2 следует, что понижение размерности задачи несущественно сказывается на значениях показателей качествва прогнозирования, но, в тоже время позволяет понизить трудоемкость решения задачи.

\section{Выводы}

Разработана программа решения задачи прогнозирования методом градиентного бустинга на базе обучающей выборки организованной в виде большого массива данных. Исследование возможности использования других методов машинного обучения для задач данного класса проведено в работе [3].

Проведен анализ возможности понижения размерности обучающей выборки с целью сокращения трудоемкости решения задачи без потери информации, содержащейся в массиве исходных данных.

\section{Список литературы}

1. Ким Н.Г. Прогнозирование котировок ценных бумаг методами линейной регрессии, дерева решений и с помощью многослойной нейронной сети / Н.Г. Ким, Л.Д. Хлебородова // Студент года 2021: Сборник статей Международного учебно-исследовательского конкурса в 6-ти частях, 
Петрозаводск, 19 мая 2021 года. - Петрозаводск, 2021. - С. 288-292. - DOI 10.46916/02062021-4-978-5-00174-249-4.

2. А. Дьяконов «Введение в анализ данных и машинное обучение». URL: https://alexanderdyakonov.files.wordpress.com/2017/06/book_boosting_ pdf.pdf (Дата обращения 29.10.2021).

3. Хлебородова Л.Д. Сравнение методов машинного обучения для задачи прогнозирования в среде Wolfram Mathematica / Л.Д. Хлебородова, Г.С. Осипов // Постулат. № 10 (2021), с. 10.

4. Stephen Wolfram. An Elementary Introduction to the Wolfram Language. URL: https://www.wolfram.com/language/elementary-introduction/2nd-ed/ (Дата обращения 29.10.2021).

СЛ.Д. Хлебородова, Г.С. Осипов 2021 